\title{
Pénuries de médicaments : le sondage a parlé...
}

\author{
par Douglas Sellinger
}

$\mathrm{M}$ a fille sera bientôt diplômée du secondaire et vient de remplir sa demande d'admission à l'université. Elle amorcera un programme préparatoire à la pharmacie et pense faire carrière comme pharmacienne hospitalière, alors on dirait que la pomme n'est pas tombée trop loin du pommier. Je me souviens de l'émoi qu’ont créé au sein de la famille ses débuts à l'école il y a environ 12 ans. Chaque année, elle participait à de plus en plus d'activités scolaires et parascolaires. Et comme notre travail connexe en tant que parents augmentait, notre capacité à accomplir les tâches augmentait également, en partie parce que nous avions abandonné d'autres activités. À de nombreux égards, la situation des pénuries de médicaments au Canada a eu le même effet sur les pharmaciens.

La Société canadienne des pharmaciens d'hôpitaux (SCPH) a joint ses efforts à ceux de l'Association des pharmaciens du Canada et de l'Association médicale canadienne pour sonder les membres sur les pénuries de médicaments et communiquer une déclaration sur les résultats du sondage aux médias'. Si vous avez manqué la nouvelle dans votre bulletin local, ne vous en faites pas : la couverture par les médias était plutôt limitée tout comme l'intérêt de nos politiciens. Lélément qui manquait peut-être à cette histoire était le tort potentiel causé au patient. Les mesures pour pallier les pénuries de médicaments qui ont été mises de l'avant de façon rapide et opportune par les pharmaciens d'hôpitaux et communautaires ainsi que les médecins ont eu un effet positif sur de nombreux résultats cliniques pour les patients, mais ces succès ne semblent pas avoir ce qu'il faut pour faire les manchettes.

Les résultats du sondage ont indiqué que jusqu’à $20 \%$ des patients avaient été touchés par les pénuries. Les trois principales répercussions des pénuries pour les patients étaient l'accès retardé à un médicament essentiel, l'utilisation d'un médicament ou d'une forme pharmaceutique moins efficace et le risque accru d'effets indésirables. Les pharmaciens d'hôpitaux qui ont répondu au sondage ont indiqué que les patients ont dû composer avec des retards d'approvisionnement en médicaments, des coûts additionnels associés aux médicaments de rechange et une certaine confusion liée aux changements de médicament ou de forme pharmaceutique.

Des 215 pharmaciens hospitaliers qui ont répondu, plus de la moitié ont signalé des problèmes à trouver une source d'approvisionnement en médicament pour un patient durant

leur plus récent quart de travail et près des deux tiers ont indiqué avoir eu des problèmes à obtenir un médicament dans la semaine précédente. Pour atténuer les effets des pénuries de médicaments, les pharmaciens hospitaliers avaient recours à diverses stratégies, notamment : communiquer avec les collègues de la pharmacie, de l'hôpital et de la régie régionale de la santé; rationner ou restreindre l'emploi des approvisionnements existants; obtenir des médicaments d'une autre source; préparer des médicaments à partir d'une concentration ou d'une forme pharmaceutique différente d'un même médicament; changer de voie d'administration; ou recourir à un autre médicament ne faisant pas l'objet d'une pénurie. Toutes ces stratégies exigent du temps, souvent du temps des pharmaciens et souvent au détriment d'autres tâches, dont la prestation des soins directs aux patients.

On a assisté à un changement de paradigme dans le réseau de la santé où les pénuries de médicaments font partie du quotidien, et les pharmaciens hospitaliers sont parmi les premiers exposés à ce changement. Des occasions de créer des stratégies efficaces et cliniquement appropriées d'utilisation des médicaments en situation de pénurie et en situation normale s'offrent donc à nous. Les patients comptent sur notre expertise en gestion des médicaments et sur notre compassion pour les aider à traverser cette période sombre de pénuries de médicaments.

Bien que la SCPH comprenne que les pénuries de médicaments exigent une solution à l'échelle mondiale, elle travaille ici au Canada de concert avec de multiples intervenants, dont les gouvernements fédéral, provinciaux et territoriaux, pour réduire la survenue de ces pénuries de médicaments et pour atténuer leurs effets sur les patients et les professionnels de la santé. Grâce à ces efforts, j'espère que la SCPH pourra réduire les répercussions des pénuries de médicaments et soulager le stress et l'anxiété qu'elles suscitent aux pharmaciens d'hôpitaux et à leurs patients.

[Traduction par l'éditeur]

\section{Reference}

1. Canadians paying the price for drug shortages: survey [communiqué de presse]. Ottawa (ON) : Canadian Pharmacists Association; 14 janvier 2013. Publié au www.pharmacists.ca/index.cfm/news-events/news/canadians-paying-the-pricefor-drug-shortages-survey/. Consulté le 25 février 2013.

Douglas Sellinger, BSP, MALT, est président et agent de liaison externe pour la Société canadienne des pharmaciens d'hôpitaux. 